ONLINE MUTATION REPORT

\title{
Homozygosity for autosomal dominant facioscapulohumeral muscular dystrophy (FSHD) does not result in a more severe phenotype
}

\author{
M M O Tonini, R C M Pavanello, J Gurgel-Giannetti, R J Lemmers, S M van der Maarel, R R Frants, \\ M Zatz
}

E acioscapulohumeral muscular dystrophy (FSHD) is a relatively common autosomal dominant disorder with an estimated incidence of 1:20 000 births. $^{1}$ It is clinically characterised by progressive weakness of facial, shoulder girdle, and upper arm muscles; less frequently, the lower limbs are also affected. Some intriguing phenotypic observations are still not understood at the molecular level, such as the existence of clinical anticipation in some multigenerational families, which was observed in several population studies. ${ }^{2-5}$ In addition, a remarkable inter- and intra-familial clinical variability, ranging from severe infantile forms to asymptomatic or non-penetrant gene carrying, may occur in individuals with an FSHD deleted fragment of the same size. It has been reported that a greater proportion of females than males remain asymptomatic, ${ }^{16}$ and more recently we have observed that these non-penetrant individuals seem to be concentrated in some families. ${ }^{7}$

The FSHDI locus has been mapped to chromosome 4q35, and in most patients the probe pl3E-11 detects a polymorphic EcoRI fragment smaller than $35 \mathrm{~kb}$. The size of this fragment ranges from 35 to $300 \mathrm{~kb}$ in normal individuals and consists of multiple copies of a tandem repeated $3.3 \mathrm{~kb} K p n \mathrm{I}$ unit (D4Z4). ${ }^{8}$ As the $4 \mathrm{q} 35$ region is highly homologous to $10 \mathrm{q} 26$, the confirmation of molecular diagnosis is performed using $B \ln \mathrm{I}$, which cleaves the $10 \mathrm{q} 26$ units only into the $3.3 \mathrm{~kb}$ fragments, which are undetectable. ${ }^{9}$ It has been shown that deletions of an integral number of the chromosome 4 units lead to FSHD and that this contraction might affect nearby genes by altering the chromosomal structure, inducing position effect variegation. ${ }^{10}$

A polymorphic segment distal to D4Z4 with alleles 4qA and $4 \mathrm{qB}$ was described by Van Geel et al. ${ }^{11}$ In a survey in the Dutch population, Lemmers et $a l^{12}$ found that while in control individuals both alleles are equally present, FSHD is uniquely associated with the $4 \mathrm{qA}$ allele. Moreover only type A alleles were observed on chromosome 10.

Gabellini et al $^{13}$ described a repressor complex that binds to the D4Z4 repeats. Partial deletion of this repeat would result in transcriptional derepression and consequently upregulation of the genes proximal to $4 \mathrm{q} 35$. According to these authors, the overexpression is inversely related to the number of D4Z4 repeats, which would explain why patients with a severe course have on average the smallest EcoRI fragments. Yip et $a l^{14}$ examined the effect on reporter gene activity and myoblast differentiation in vitro of increasing the D4Z4 repeat number, and observed that increasing D4Z4 repeats have a significant trans effect on myoblast differentiation with only a minor cis effect on reporter gene activity.

Although FSHD is an autosomal dominant condition, asymptomatic or mildly affected individuals may not be diagnosed, and consequently offspring from two FSHD parents may carry two deleted alleles. Although individuals

\section{Key points}

- Facioscapulohumeral muscular dystrophy (FSHD) is a relatively common autosomal dominant disorder, characterised by progressive weakness of the facial, shoulder girdle, and upper arm muscles, and less frequently, by weakness in the lower limbs.

- We report a 29 year old patient with FSHD, who presented to our centre. This patient is a member of a large, inbred family, several members of which also showed clinical signs of FSHD.

- We examined 36 individuals from this family; four, including the proband, were found to be clinically affected, with two others showing some abnormal signs on neurological examination.

- Molecular analysis showed that 17 of the 36 members carried a $24 \mathrm{~kb}$ EcoRI fragment, indicating deletions in the FSHD allele. Although the proband was homozygous for this allele, he was not more severely affected than heterozygous members. In fact, his very severely affected cousin did not carry this deletion, and the cause of her condition is still unknown.

- We describe what we believe to be the first homozygous case of FSHD, and show that the homozygous state is not only compatible with life, but does not necessarily result in a worse clinical presentation. Further analysis of this family should help in the understanding of FSHD.

homozygous for other autosomal dominant diseases have been reported, this condition has never been described for FSHD.

In Huntington's Disease (HD), an autosomal dominant late onset disorder, homozygous individuals were described in the pre-molecular era ${ }^{16}$ and confirmed at a later stage through molecular analysis. ${ }^{16}$ Surprisingly, not only was the age at onset not earlier in homozygous compared with heterozygous patients, but in fact, heterozygous individuals seemed to have a more rapid progression of disease than homozygotes. ${ }^{17}$ On the other hand, there are some diseases where homozygotes seem to be more severely affected than heterozygotes. One

Abbreviations: $\mathrm{CK}$, creatinine kinase; FSHD, facioscapulohumeral muscular dystrophy; HD, Huntington's disease; PFGE, pulsed field gel electrophoresis 
such disease is Machado-Joseph disease (SCA3), which is surprising because the pathophysiological mechanism (expansion of the polyglutamine tract) is the same as in HD. ${ }^{18}$

Here we report the first case to our knowledge of homozygosity in an FSHD patient with a classical phenotype, who inherited two identically sized FSHD alleles from a common ancestor. This patient was ascertained in a very large white Brazilian inbred family with very interesting features. In addition to several non-penetrant FSHD carrier relatives, the proband also has some relatives presenting the classical FSHD phenotype, and a severely affected female cousin. The study of this patient demonstrates that not only is homozygosity for FSHD compatible with life, but most interestingly, that it does not result in a more severe phenotype.

\section{PATIENTS AND METHODS \\ Patients}

The proband, a 29 year old Brazilian male (IV-11, fig 1), was referred to the Human Genome Research Center at the Department of Biology, University of São Paulo, with a clinical diagnosis suggestive of FSHD. He reported onset at 16 years of age when he noticed difficulties with carrying weight. At presentation, he showed proximal atrophy, upper limb muscle weakness, and winged scapulae, but no facial weakness. His lower limb muscles were spared. His serum creatine kinase (CK) was increased approximately twofold above normal.

The proband belongs to a heavily inbred genealogy and his parents were first degree cousins. On the first consultation, he mentioned having some affected relatives (individuals IV1 and IV-14, fig 1), including a severely affected, wheelchair bound, 26 year old female cousin (IV-7).

The proband's family members came to our centre for examination or were visited at home. A total of 36 individuals (21 females and 15 males) (fig 1 ), with ages ranging from 21 to 81 years old were analysed. They were submitted to careful clinical and neurological examination, and blood was drawn for serum CK determination and molecular analysis. The clinical classification of family members as affected or non-affected was performed by the neurologist, prior to the information on molecular analysis results. All subjects gave informed consent.

On clinical examination, we observed that the 53 year old mother of the proband (III-12) was clinically affected. She presented a mild course with upper limb muscle weakness. The father (III-13) had died at the age of 46 years in an accident but was reported as normal. The proband's sister (IV-10) and two brothers (IV-12 and IV-13) presented no abnormalities at clinical and neurological examination. However, the youngest brother (IV-14), aged 18 years, presented winged scapulae, discrete weakness in upper limb muscles, facial weakness, and general muscle atrophy. His serum CK was in the normal range.

A cousin, IV-1, aged 31 years, presented facial, upper limb, and mild lower limb weakness, which she reported as having since childhood. Her serum CK was slightly increased (1.5 fold above normal). The other cousin of the proband, IV-7, has been wheelchair bound since the age of 12 years. Her serum CK showed normal levels on two independent samples. According to her mother, she started walking at 2 years of age with onset of muscular weakness in the lower limbs. Currently, at 29 years, she presents abolished reflexes, and severe general muscle atrophy and weakness in the lower and upper limbs, and is unable to raise up both her arms simultaneously. In addition, she has alopecia and shows signs of premature ageing, but has no facial or cognitive involvement.
Five individuals from the second generation (individuals II-4 to II-8, aged 73 to 81 years), 16 from generation III and 12 from generation IV, were also examined. All were classified as clinically normal with the exception of two members, who had minimal signs on clinical/neurological examination. Subject III-20, aged 51 years, considered himself as normal but showed pectus escavatum and an almost imperceptible facial weakness when carefully examined. Subject III-16, aged 32 years, had hyporeflexia on neurological examination but no apparent clinical signs.

\section{METHODS}

DNA extraction from whole blood, digestion with EcoRI and EcoRI-BlnI, linear electrophoresis, and hybridisation with probe pl3E-11 were performed as described elsewhere. ${ }^{8}$ The same procedure was used for pulsed field gel electrophoresis (PFGE). ${ }^{18}$ After hybridisation, the PFGE membranes were exposed to Phosphorimager screens and analysed with the ImageQuant software program (Molecular Dynamics).

The dosage test ${ }^{19}$ is based on the digestion of liquid DNA with $B g / \mathrm{II} / B \ln \mathrm{I}$ and subsequent hybridisation of conventional Southern blots with pl3E-11. Analysis was also performed based on intensity of the signals using the Phosphorimager screens and ImageQuant software. This is a useful technique, which can help in elucidating the allelic constitution of an individual when PFGE is not available or uninformative.

In order to search for a recombination that could distinguish between the two chromosome 4 alleles, we looked at the family's haplotype using two markers proximal to the D4Z4 repeat: D4S139 and D4S163 (fig 1). We digested $5 \mu \mathrm{g}$ of genomic DNA with PstI, ran this overnight in a $0.7 \%$ agarose gel, and hybridised with the Lila 5 probe to analyse locus D4S163. The same procedure was repeated with TaqI and probe PH30 for locus D4S139.

To analyse the distal part of $4 \mathrm{q}$ for the $4 \mathrm{qA}$ or $4 \mathrm{qB}$ alleles we used probes specific for HindIII fragments of these two alleles. ${ }^{12}$

\section{RESULTS}

We first tested the proband and his mother (III-12) and observed that both of them carried a deleted $24 \mathrm{~kb} F S H D$ fragment that was also found in the affected cousin (IV-1) and affected brother (IV-14). Surprisingly, the proband's sister (IV-10, aged 31 years) and one of his brothers (IV-12, aged 25 years), who were considered normal following neurological examination, had also inherited the FSHD allele. Subsequently, after collecting blood and examining the rest of the family, we performed a PFGE analysis and observed that the intensity of the $24 \mathrm{~kb} E c o$ RI fragment of the proband was stronger than those observed in other relatives (fig 2), indicating that the patient had two chromosome 4 alleles of the same size $(24 \mathrm{~kb})$.

The $24 \mathrm{~kb}$ EcoRI fragment was found in 17 (9M, 8F) of 36 tested individuals, and therefore was segregating according to autosomal dominant inheritance. However, only four members of this family (the 29 year old proband, and his 18 year old brother, 53 year old mother, and 31 year old female cousin) were considered clinically affected, while two additional members (one male, III-20, and one female, III-16, aged 51 and 32 years, respectively) had some abnormal signs identified during neurological examination. Unexpectedly, the severely affected cousin IV-7 did not carry the FSHD deleted allele, nor was this found in either of her parents. Therefore, the cause of her neuromuscular disorder is still unknown.

The large proportion of asymptomatic or only minimally affected individuals in this family raised the question whether the FSHD allele segregating in this family was pathogenic. The hybridisation with probes specific to $4 \mathrm{qA}$ and 

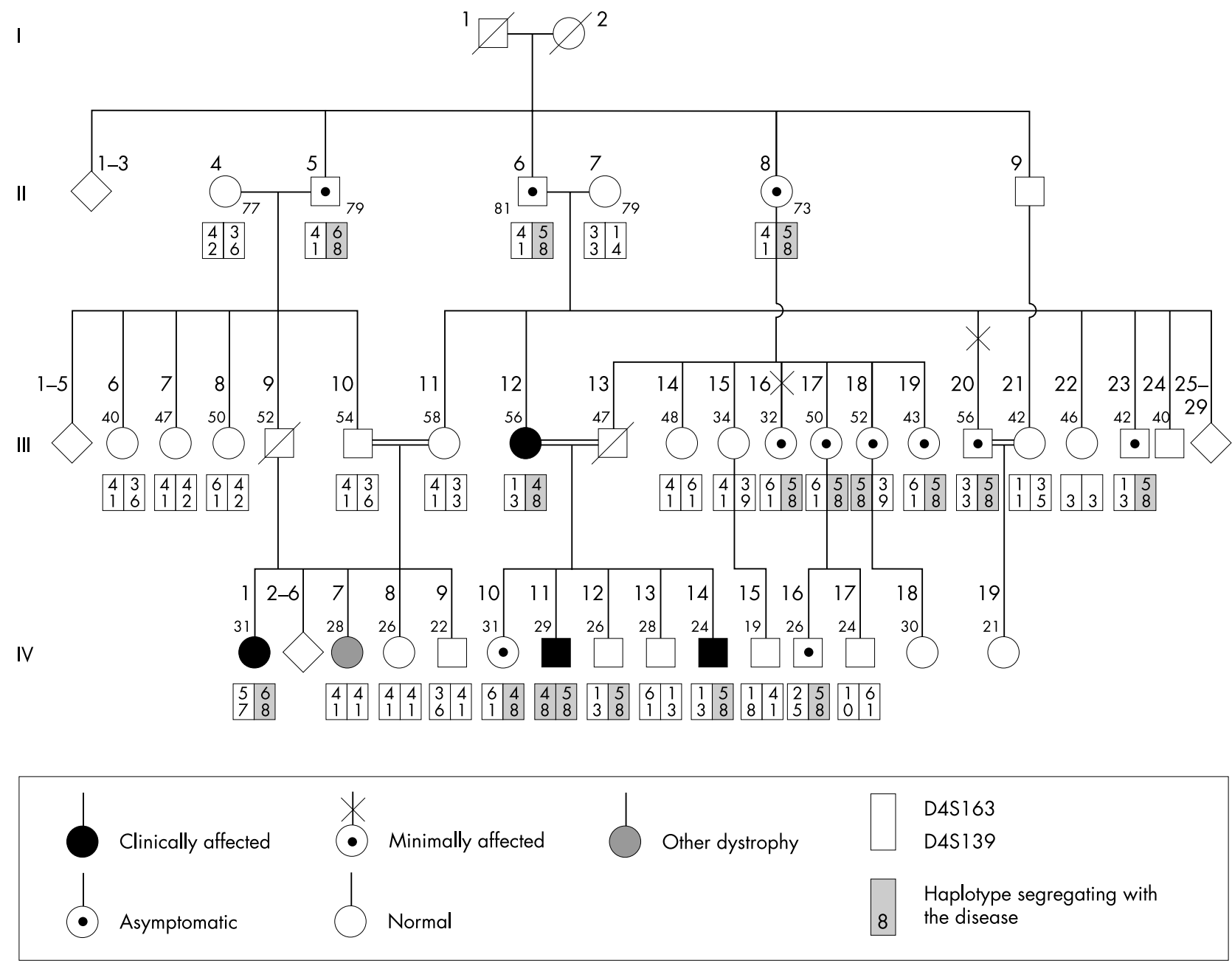

Figure 1 Pedigree showing the examined and molecularly analysed individuals. Subject IV-11 is homozygous for the FSHD deleted allele, confirmed through haplotype analysis, while his affected brother IV-14 inherited just one allele. Siblings IV-10 and IV-12 are asymptomatic. Subject IV-7 is severely affected by another neuromuscular disorder but did not inherit the FSHD deleted fragment.

$4 \mathrm{qB}$ showed that the FSHD allele was of the 4qA type in all individuals as well as in the homozygous proband, who carried two 4qA alleles (fig 3).

In addition, the family's haplotype was analysed in an attempt to identify any difference between the two alleles that could explain the observed clinical variability (fig 1). Analysis with marker D4S139, the most distal of both markers, showed that all the individuals with the FSHD deletion shared allele 8 , including the homozygous proband, who proved to be homozygous for this marker. When D4S163, the most proximal marker, was analysed, we could observe that two different alleles, 5 and 6, were segregating among members from generation II, but the segregation was consistent with the presence of the deleted fragment. A recombination event was identified during the transmission from individual II-6 to III-12 (the only one who carries allele 4 and the deleted fragment). Individual III-12 transmitted the 4,8 haplotype to her asymptomatic daughter and to the homozygous proband. The affected brother (IV-14), as well as the asymptomatic individuals from the second and third generations, inherited the 5,8 haplotype.

\section{DISCUSSION}

FSHD is usually a relatively mild condition, although with a highly variable phenotype. Thus, the fact that no individual homozygous for this condition has been reported previously suggests either that the disease does not occur sufficiently frequently for homozygotes to be produced, or that this condition is not compatible with life. Although rare, the existence of large inbred families such as this one, with several affected members, allows the mating of two FSHD carriers and consequently the birth of individuals homozygous by common origin. The analysis of individuals from these genealogies might bring new insights in the pathogenesis of this complicated condition and help the understanding of the clinical variability in individuals carrying the same mutation.

Compound heterozygosity has been recently described ${ }^{20}$ in two Dutch FSHD families, showing that this condition is compatible with life in both males and females. In the first family, the severely affected proband carries a $17 \mathrm{~kb}$ and a $24 \mathrm{~kb}$ fragment. In the second family, the proband, who carries one $33 \mathrm{~kb}$ and one $36 \mathrm{~kb}$ allele, presents a classical phenotype. The authors suggest a possible dosage effect in the probands in which each allele contributed to the FSHD phenotype. However, because in these two families the probands are compound heterozygotes for two differently sized alleles, it is more difficult to assess the phenotypic effect of each of the alleles in the individuals found to carry both of them. We were able to address this question in our Brazilian family because our proband carries two identically sized deletion alleles inherited from a common ancestor. It is 


\section{$\begin{array}{lllllllll}1 & 2 & 3 & 4 & 5 & 6 & 7 & 8 & 9\end{array}$}

$\mathrm{E} / \mathrm{H} \mathrm{E} / \mathrm{B} \mathrm{E} / \mathrm{H} \mathrm{E} / \mathrm{B} \mathrm{E} / \mathrm{H} \mathrm{E} / \mathrm{B} E / \mathrm{H} \mathrm{E} / \mathrm{H} \mathrm{E} / \mathrm{B}$

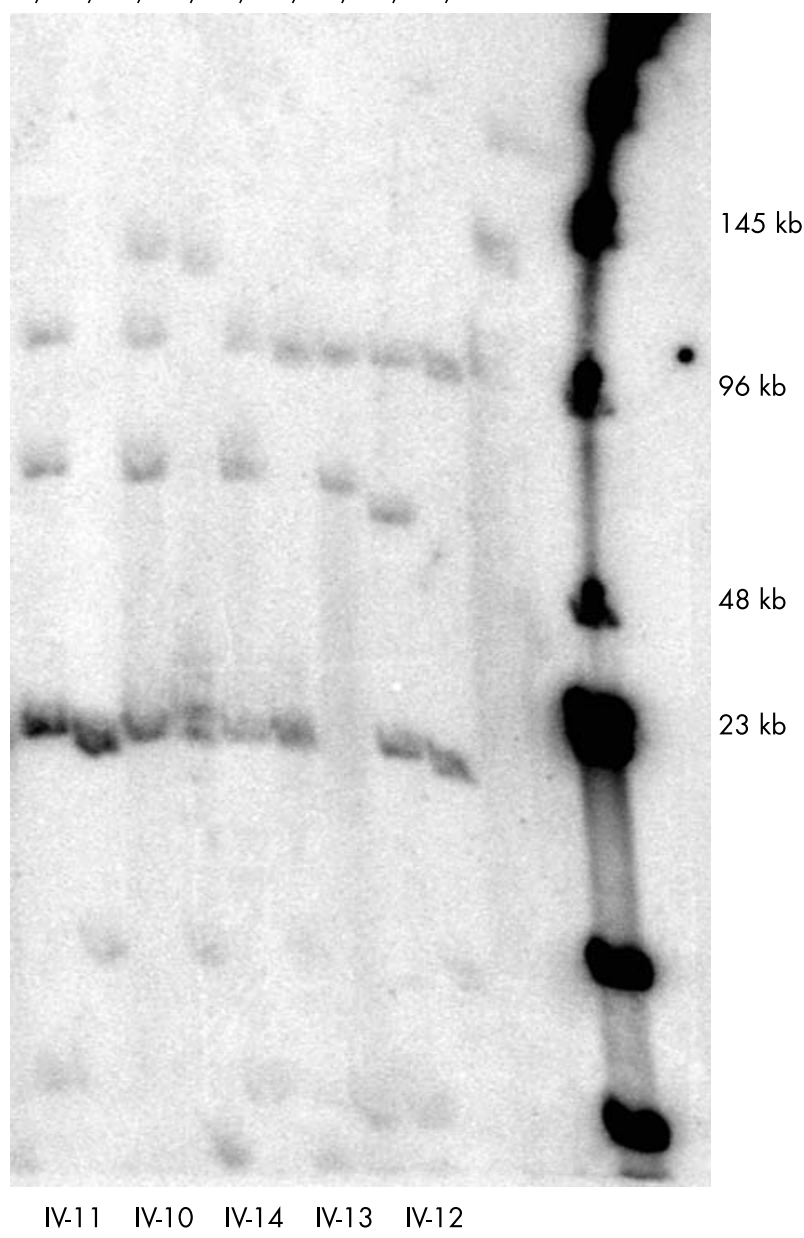

Figure 2 PFGE (pulsed field gel electrophoresis) showing double digestion with enzymes EcoRl/Hindlll $(E / H)$ and EcoRl/Blnl (E/B) performed in all subjects except IV-13, whose DNA was digested only with EcoRl/Hindlll. The gel was blotted and hybridised with p13E-11. The stronger fragment (lanes 1,2) indicates that the proband (IV-11) carries two deleted fragments inherited both from his mother and father. He also has two chromosome 10 alleles of approximately 60 and $100 \mathrm{~kb}$. Individual IV-10 (lanes 3,4), who is the asymptomatic sister, carries the $24 \mathrm{~kb}$ and $60 \mathrm{~kb}$ alleles from chromosome 4 , and the $100 \mathrm{~kb}$ and $130 \mathrm{~kb}$ alleles from chromosome 10. The affected brother (IV-14, lanes 5,6) carries the FSHD allele as well as a $60 \mathrm{~kb}$ chromosome 10 allele and the $100 \mathrm{~kb}$ chromosome 4 allele. The other chromosome 10 allele is either co-migrating with one of the other alleles or is too large to be detected in the gel. Subject IV-13 (lane 7) does not carry the FSHD allele. Subject IV-12 (lanes 8, 9), the asymptomatic brother, carries the $24 \mathrm{~kb}$ and $100 \mathrm{~kb}$ chromosome 4 alleles and a $55 \mathrm{~kb}$ chromosome 10 allele. As in individual IV-4, the other chromosome 10 allele could not be visualised.

noteworthy that his phenotype is not more severe than the average FSHD phenotype even though he carries two $24 \mathrm{~kb}$ fragments. In addition, this unique family provides an answer to several other speculative hypotheses.

- The possibility that one of the short alleles would not be pathogenic. However, as the proband's parents were first cousins, it is unlikely that he inherited different alleles from both his parents. In addition, both the 4-8-24 and the $5-8-24$ alleles were observed to be pathogenic in his relatives.

- The possibility that the $4 \mathrm{qB}$ and not the $4 \mathrm{qA}$ allele was segregating in this family. In this case, the phenotype of the propositus might be the result of two "non-pathogenic" 4qB fragments. This hypothesis would also explain the large proportion of asymptomatic carriers in this family-that is, they would be carrying the $4 \mathrm{qB}$ and not the $4 \mathrm{qA}$ allele. However, this possibility was ruled out as all the short alleles in the family members were found to be $4 \mathrm{qA}$.

- The observation that two deleted fragments did not result in a more severe phenotype in our proband could be due to the fact that as there are many asymptomatic carriers in this family, the dosage effect of two "non-penetrant" 4qA alleles could result in a classical phenotype. Indeed, the proband (IV-11) is more severely affected than his mother (III-12). However, his cousin (IV-1) and his 18 year old brother (IV-14), both with a classical course, have just one deleted allele, indicating that this is in fact sufficient to cause the disease. Therefore, the observation that the two brothers are more severely affected than their mother is probably due to clinical anticipation, ${ }^{2}$ although the possibility of other modifying loci or epigenetic factors should also be considered.

- The possibility that different haplotypes could be subject to different activation by the D4Z4 binding element, considered to be a transcriptional repressor element. ${ }^{13}$ However, the segregation of two analysed markers from the $4 \mathrm{q} 35$ region (alleles 4,5 , and 6 from marker D4S163 and allele 8 from marker D4S139) showed no apparent association with the phenotype, as the affected proband's brother inherited the same haplotype as his asymptomatic paternal uncles and aunts. Moreover, the proband's asymptomatic siblings inherited different haplotypes, one from the mother and the other from the father.

The large proportion of "non-penetrant" cases in this genealogy also caught our attention and supports our observation that asymptomatic carriers seem to be concentrated in some families. ${ }^{7}$ It is unlikely that this large proportion of asymptomatic individuals is due to the fragment size $(24 \mathrm{~kb})$, as asymptomatic carriers are more often reported among individuals carrying larger borderline fragments (larger than $33 \mathrm{~kb}$ ). Interestingly, we had previously observed in other families with non-penetrant cases that they carried a deleted fragment of a similar size $(23 \mathrm{~kb}){ }^{6}$

Finally, the observation that individual IV-7, wheelchair bound since 12 years of age, did not carry the FSHD fragment was surprising as our first hypothesis was that she might be a homozygous FSHD carrier. The cause of her disorder is still unknown, but could be a recessive condition due to parental consanguinity. However, it is of interest that we have already described the existence of two different forms of dystrophy in two unrelated non-consanguineous families with FSHD patients. ${ }^{21}$

In short, there are three intriguing findings in this family that await an explanation at the molecular level: $(a)$ the existence of two unrelated disorders in the same family; $(b)$ the observation that homozygous FSHD does not necessarily result in a more severe phenotype; and $(c)$ the large proportion of asymptomatic carriers.

It will be of great interest to investigate gene expression levels in both homozygous and asymptomatic individuals in light of the transcriptional derepression model, which considers upregulation of genes such as FRG2 to be inversely related to the size of the deletion. ${ }^{13}$ However, it is difficult to explain under this hypothesis why individuals carrying the same deleted allele may be clinically affected or asymptomatic. It is still more intriguing that our homozygous proband with two deleted FSHD alleles is not more severely affected than his brother, who carries only one deleted allele. 




Figure 3 PFGE of the DNA of 27 individuals, which was digested with Hindlll and hybridised with $4 \mathrm{qA}$ probe to confirm that all deleted chromosome 4 alleles were from the $4 \mathrm{qA}$ type. The DNA from all subjects considered to have an FSHD fragment hybridised to the $4 \mathrm{qA}$ probe. Each lane represents one family member whose position in the pedigree (fig 1) is shown at the bottom of the gel.
Finding the mechanism that modulates the clinical severity and in particular, that protects some individuals from the pathogenic effect of the deletion could shed light on this intriguing disease, which seems to become more and more complex.

\section{ACKNOWLEDGEMENTS}

The collaboration of the following persons is gratefully acknowledged: A Cerqueira, A Nishimura, K Abe, F de Paula, Dr M R PassosBueno and C Urbani. Special thanks to all patients and relatives for their cooperation. This work is supported by grants from FAPESPCEPID, CNPq, PRONEX, CAPES.

\section{Authors' affiliations}

M M O Tonini, R C M Pavanello, M Zatz, Human Genome Research Centre, Departamento de Biologia, Instituto de Biociencias, Universidade de São Paulo, São Paulo, Brazil

J Gurgel-Giannetti, Division of Neuromuscular Diseases, Hospital das Clínicas, Universidade Federal de Minas Gerais, MG-Brasil

R J Lemmers, S M van der Maarel, R R Frants, Centre for Human and Clinical Genetics, Leiden University Medical Centre, Leiden, The Netherlands

Correspondence to: Professor M Zatz, Centro de Estudos do Genoma Humano, Departamento de Biologia, Instituto de Biociencias,
Universidade de São Paulo, São Paulo, 05508-900, Brazil; mayazatz@ib.usp.br

\section{REFERENCES}

1 Padberg GW. Facioscapulohumeral disease. PhD Thesis. Leiden: Leiden University, 1982:243.

2 Zatz M, Marie SK, Passos-Bueno MR. High proportion of new mutations and possible anticipation in Brazilian facioscapulohumeral muscular dystrophy families. Am J Hum Genet 1995;56:99-105.

3 Lunt PW, Jardine PE, Koch MC, Maynard J, Osborn M, Williams M, Harper PS, Upadhyaya M. Correlation between fragment size at D4F104S1 and age of onset or at wheelchair use, with a possible generational effect, account for much phenotypic variation in 4q35-facioscapulohumeral muscular dystrophy (FSHD). Hum Mol Genet 1995;4:951-8.

4 Goto K, Lee JH, Matsuda C, Hirabayashi K, Kojo T, Nakamura A, Mitsunaga Y, Furukawa T, Sahashi K. Arahata K DNA rearrangements in Japanese facioscapulohumeral muscular dystrophy patients: clinical correlations. Neuromuscul Disord May 1995;5:201-8.

5 Tawil R, Forrester J, Griggs RC, Mendell J, Kissel J, McDermott M, King W, Weiffenbach B, Figlewicz D. Evidence for anticipation and association of deletion size with severity in facioscapulohumeral muscular dystrophy. Ann Neurol 1996;39:744-8.

6 Zatz M, Marie SK, Cerqueira A, Vainzof M, Pavanello RC, Passos-Bueno MR. The facioscapulohumeral muscular dystrophy (FSHD1) affects males more severely and more frequently than females. Am J Med Genet 1998;77:155-61.

7 Tonini MMO, Passos-Bueno MR, Cerqueira A, Matioli SR, Pavanello R, Zatz M. Asymptomatic carriers and gender differences in facioscapulohumeral muscular dystrophy (FSHD). Neuromuscul Disord 2004;14:33-8. 
8 Wijmenga C, Hewitt JE, Sandkuiil LA, Clark LN, Wright TJ, Dauwerse HG Gruter AM, Hofker MH, Moerer P, Williamson R, van Ommen GJ, Padberg GW, Frants RR. Chromosome 4q DNA rearrangements associated with facioscapulohumeral muscular dystrophy. Nat Genet 1992;2:26-30.

9 Deidda G, Cacurri S, Piazzo N, Felicetti L. Direct detection of $4 \mathrm{q} 35$ rearrangements implicated in facioscapulohumeral muscular dystrophy (FSHD). J Med Gen 1996;33:361-5.

10 Winokur ST, Bengtsson U, Feddersen J, Mathews KD, Weiffenbach B, Bailey H, Markovich RP, Murray JC, Wasmuth JJ, Altherr MR, Schutte BC. The DNA rearrangement associated with facioscapulohumeral muscular dystrophy involves a heterochromatin associated repetitive element: implications for a role of chromatin strucuture in the pathogenesis of the disease. Chromosome Res May 1994;2:225-34.

11 Van Geel M, Dickson M, Beck A, Bolland D, Frants RR, van der Maarel S, de Jong P, Hewitt J. Genomic analysis of human chromosome $10 q$ and $4 q$ telomeres suggests a common origin. Genomics 2002;79:210-17.

12 Lemmers RJ, de Kievit P, Sandkuij L, Padberg GW, van Ommen GJ, Frants RR, van der Maarel SM. Facioscapulohumeral muscular dystrophy is uniquely associated with one of the two variants of the $4 \mathrm{q}$ telomere. Nature Genetics 2002;32:1-2

13 Gabellini D, Green M, Tupler R. Inappropriate gene activation in FSHD: a repressor complex binds a chromosomal repeat deleted in dystrophic muscle. Cell 2002;110:339-48.

14 Yip DJ, Picketts DJ. Increasing D4Z4 repeat copy number compromises C2C12 myoblast differentiation. FEBS Lett 2003;537:133-8.
15 Wexler NS, Young AB, Tanzi RE, Travers H, Starosta-Rubinstein S, Penney JB, Snodgrass SR, Shoulson I, Gomez F, Ramos Arroyo MA, Penchaszadek GK, Moreno H, Gibbons K, Faryniarz A, Hobbs S, Anderson MA, Bonilla E, Conneally PM, Gusella JF. Homozygotes for Huntington's disease. Nature 1987;326:194-7.

16 Durr A, Hahn-Barma V, Brice A, Pecheux C, Dode C, Feingold J. Homozygosity in Huntington's disease. J Med Genet 1999;36:172-3.

17 Zlotogora J. Dominance and homozygosity. Am J Med Genet 1997;68:412-16.

18 Van Deutekom JCT. Towards the molecular mechanism of facioscapulohumeral muscular dystrophy. PhD thesis. Leiden: University of Leiden, 1996.

19 Van der Maarel S, Deidda G, Lemmers R, Bakker E, van der Wielen M, Sandkuijl L, Hewitt J, Padberg G, Frants R. A new dosage test for subtelomeric 4;10 translocations improves conventional diagnosis of facioscapulohumeral muscular dystrophy. J Med Genet 1999;36:823-8.

20 Wohlgemuth $M$, Lemmers RJ, van der Kooi EL, van der Wielen MJ, van Overveld PG, Dauwerse H, Bakker E, Frants RR, Padberg GW, van der Maarel SM. Possible phenotypic dosage effect in patients compound heterozygous for FSHD-sized 4q35 alleles. Neurology 2003;14:61.

21 Tonini MM, Passos-Bueno MR, Cerqueira A, Pavanello R, Vainzof M, Dubowitz V, Zatz M. Facioscapulohumeral (FSHDI) and other forms of muscular dystrophy in the same family: is there more in muscular dystrophy than meets the eye? Neuromuscul Disord 2002;12:554-7. 